\title{
Baseline HbA1c in acute kidney injury after cardiac surgery
}

\author{
Xiaojie Liu ${ }^{1} \cdot$ He Dong ${ }^{1} \cdot$ Haichen Chu ${ }^{1}$
}

Received: 24 March 2021 / Accepted: 29 March 2021 / Published online: 9 April 2021

(c) Japanese Society of Anesthesiologists 2021

To the Editor:

We read with great interest the article from Seohee Lee and the colleagues [1] in which they included 3625 patients who accepted cardiac surgery. They found that intraoperative hyperglycemia in patients with an elevated preoperative CRP level was significantly related to an increased risk of AKI after cardiac surgery.

There are many factors affecting postoperative acute kidney injury including age, diabetes mellitus, preoperative creatinine concentration, etc. [2].

Previous studies found that glucose fluctuations are related to oxidative stress, endothelial dysfunction, and inflammation, the pathogenesis of vascular damage. However, in addition to sustained hyperglycemia, glucose fluctuations also contribute significantly to endothelial impairment. And the level of HbA1c was used to illustrate this more accurately [3, 4]. Hence, we believe that the level of $\mathrm{HbAlc}$ of the groups should be included in the baseline variable and wonder whether this factor of groups is similar and unbiased.

Funding The study was supported by excellent project of Hospital Youth Fund Grant No(2020-09) and Clinical Medicine +X Project of Qingdao UniversityGrant No(QDFY+X2021036).

This comment refers to the article available online at https://doi. org/10.1007/s00540-020-02849-w.

Haichen Chu

chiefchu@163.com

Xiaojie Liu

mmliuxiaojie@163.com

He Dong

donghedong@126.com

1 Department of Anestheiology, The Affiliated Hospital of Qingdao University, No. 59, Haier Road, Qingdao, Shandong province, China

\section{Declarations}

Conflict of interest The authors declare that they have no conflict of interest.

\section{References}

1. Lee S, Nam S, Bae J, Cho Y, Jeon Y, Nam K. Intraoperative hyperglycemia in patients with an elevated preoperative C-reactive protein level may increase the risk of acute kidney injury after cardiac surgery. J Anesth. 2021;35(1):10-19.

2. Parolari A, Pesce LL, Pacini D, Mazzanti V, Salis S, Sciacovelli C, Rossi F, Alamanni F, Monzino Research Group on Cardiac Surgery Outcomes. Risk factors for perioperative acute kidney injury after adult cardiac surgery: role of perioperative management. Ann Thorac Surg. 2012;93(2):584-91.

3. Monnier L, Mas E, Ginet C, Michel F, Villon L, Cristol J, Colette C. Activation of oxidative stress by acute glucose fluctuations compared with sustained chronic hyperglycemia in patients with type 2 diabetes. JAMA. 2006;295:1681-7

4. Škrha Jan, Šoupal Jan, Škrha Jr J, Prázný M. Glucose variability, HbA1c and microvascular complications. Rev Endocr Metab Disord. 2016;17(1):103-10.

Publisher's Note Springer Nature remains neutral with regard to jurisdictional claims in published maps and institutional affiliations. 